\title{
Storage artefacts in peripheral blood smears
}

\author{
Shaista Choudhary ${ }^{1}$, Ravi Shankar Katkar,", Divya Nagaram³ \\ ${ }^{\mathbf{1}}$ Associate Professor, ${ }^{2}$ Assistant Professor, ${ }^{3}$ Post Graduate, Dept. of Pathology, Dr. B.R. Ambedkar Medical College \& Hospital, \\ (Rajiv Gandhi University Health Science), Bangalore, Karnataka, India
}

*Corresponding Author:

Email: ravishankarkatkar@gmail.com

\begin{abstract}
Introduction: Microscopic evaluation of peripheral blood smear is a very important investigation. Blood for various laboratory analyses are commonly kept in ethylene diamine tetra acetic acid (EDTA). EDTA can however cause morphological and fragility changes in blood cells. However, morphological analyses can be greatly hampered due to occurrence of artefacts.

Aims and Objectives: To identify the storage artefacts and thus avoid misinterpretation of peripheral blood smears.

Materials and Methods: 50 blood samples were obtained using EDTA and 3.5\% sodium citrate as anticoagulants. Smears were made immediately as well as after 2, 4 and $6 \mathrm{hrs}$ of storage at room temperature. Smears obtained from same patients by finger prick method served as controls.

Conclusion: EDTA has been recommended as the anticoagulant of choice for peripheral smear but should be examined within $1 \mathrm{hr}$ of collection to avoid misinterpretation as pathological findings leading to wrong diagnosis.
\end{abstract}

Keywords: Peripheral smears, Anticoagulant, Platelets, Neutrophils, Artifacts.

\section{Introduction}

Examination of blood smears and hematologic parameters is often the first step in assessment of hematologic function and diagnosis of related diseases. Blood is collected by venipuncture into collection tubes containing anticoagulant. ${ }^{1}$ The most commonly used anticoagulants are tripotassium or trisodium salts of ethylene diamine tetra acetic acid, trisodium citrate and heparin. EDTA is the preferred anticoagulant for blood counts as it produces complete anticoagulation with minimal morphological alterations of cell. EDTA acts by removing $\mathrm{Ca}^{++}$from blood by converting it from the ionized to non ionized form i.e. a chelating agent. Heparin causes leucocyte clumping and gives a faint blue coloration in the background stained with romanowsky stains. It is not suited for platelet and leucocyte counts. ${ }^{2}$ Trisodium citrate is the preferred anticoagulant for platelet and coagulation studies. ${ }^{3}$ However EDTA causes structural, biochemical and functional damage to blood cells. These artefacts are likely to be caused by a lysolecithin formation or fall in Adenosine Triphosphate (ATP) as the blood is kept for a long time. ${ }^{4}$ So the current study was undertaken to identify storage related morphological changes in blood cells so that these artefactual changes are not misinterpreted as pathologic findings.

\section{Aims and Objectives}

To identify the storage artefacts and thus avoid misinterpretation of peripheral blood smears.

\section{Materials and Methods}

This study was carried out in hematology laboratory of Dr. B.R Ambedkar Medical College and Hospital, Bangalore. Blood samples were obtained from 50 patients whose haematological parameters were within normal limits. Blood samples were collected directly into commercially prepared vacutainers containing K3 EDTA and sodium citrate as anticoagulants. The collected samples were mixed thoroughly containing correct concentration of anticoagulant and smears were made immediately as well as after 2, 4 and $6 \mathrm{hrs}$ of storage at room temperature. Smears obtained from the same patients by finger prick method served as controls. The smears were stained with Leishman stain and studied under conventional microscope for identification of artefactual changes.

Patient's Selection Criteria: The study included random selection of routine complete blood count of both OPD and IPD patients of all age groups and both the genders. Patients with no history of fever or medication and those with normal haematological parameters tested on automated hematology analyser were included in the study.

Morphological artefacts to be studied are as follows:

Nuclear Features: Lobulations, degeneration, karyolysis, vacuolations, rupture.

Cytoplasmic Features: Vacuolations, granularity, blebs, hairy projections, degranulation, rupture.

Platelets: Swelling, Aggregation.

Others: Swollen WBC's. crenated RBC's, smudge cells, abnormal staining characteristics.

\section{Results and Observation}

The present study included 50 blood samples. Smears obtained by finger prick method without any added anticoagulant served as controls. These smears showed clumping of RBC's and aggregated platelets. No other significant morphological alterations/artefacts were observed in direct smears. Smears made immediately after addition of anticoagulant did not 
show any artefactual changes. Smears from EDTA and sodium citrate showed significant morphological artefacts on storage.

Nuclear Changes: Nuclear lobulations were observed in the beginning followed by degeneration, karyolysis or pyknosis, vacuolation and rupture which was observed after 2hrs with EDTA and as early as 0hrs with citrate blood.

Cytoplasmic Changes: These included appearance of vacuoles, cytoplasmic granules, hairy projections, blebs and rupture which was observed after 2 hrs with EDTA blood but as early as 0hrs with sodium citrate.

Platelets: Swelling of platelets occurred at $2 \mathrm{hrs}$ with EDTA and $1 \mathrm{hr}$ with citrate. Platelet aggregation that is pseudo agglutination of platelets occurred at $3 \mathrm{hrs}$ which was not observed with citrate.

These changes are shown in the Table 1

Table 1: EDTA induced storage artefacts

\begin{tabular}{|l|c|c|c|c|}
\hline Duration & WBC cytoplasmic changes & $\begin{array}{c}\text { WBC Nuclear } \\
\text { changes }\end{array}$ & $\begin{array}{c}\text { Platelet } \\
\text { swelling }\end{array}$ & $\begin{array}{c}\text { Platelet } \\
\text { aggregation }\end{array}$ \\
\hline 0hrs & No change & No change & No change & No change \\
\hline $2 \mathrm{hrs}$ & Vacuoles (97\%) and rupture (40\%) & Degeneration (60\%) & $80 \%$ & $8 \%$ \\
\hline $4 \mathrm{hrs}$ & Hairy projections (80\%) & $\begin{array}{c}\text { Lobulations (97\%) and } \\
\text { vacuolation (25\%) }\end{array}$ & $85 \%$ & $25 \%$ \\
\hline $6 \mathrm{hrs}$ & Blebs and rupture (90\%) & Rupture (95\%) & $90 \%$ & $80 \%$ \\
\hline
\end{tabular}

Other Artefacts: Changes like smudge cells, swelling of WBC's, crenated RBC's and abnormal staining characters were also observed which began at 3-4hrs with EDTA blood and as early as $1 \mathrm{hr}$ with citrate blood.

These changes are shown in Table 2

Table 2: EDTA induced other storage artefacts:

\begin{tabular}{|l|c|c|c|c|}
\hline Duration & Smudge cells & Swollen WBC & Crenated RBC & Abnormal staining \\
\hline $0 \mathrm{hrs}$ & No change & No change & No change & No change \\
\hline $2 \mathrm{hrs}$ & $30 \%$ & $35 \%$ & $45 \%$ & $10 \%$ \\
\hline $4 \mathrm{hrs}$ & $12.5 \%$ & $40 \%$ & $40 \%$ & $25 \%$ \\
\hline $6 \mathrm{hrs}$ & $80 \%$ & $82 \%$ & $90 \%$ & $85 \%$ \\
\hline
\end{tabular}

Graph 1: Graphical representation of EDTA induced nuclear artefacts-

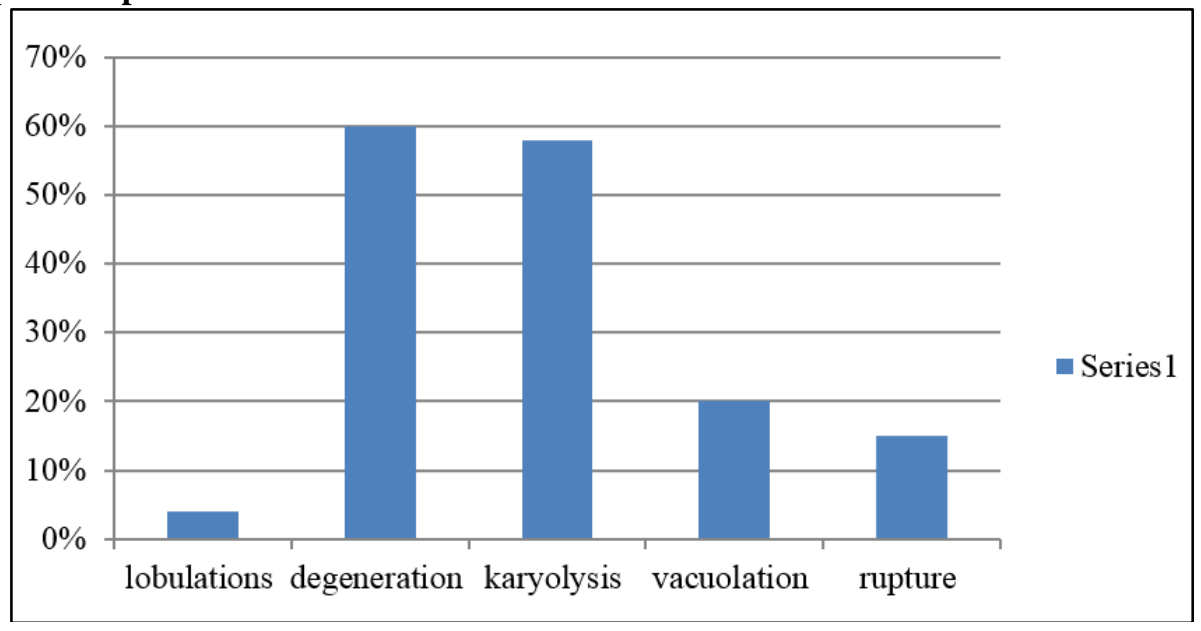




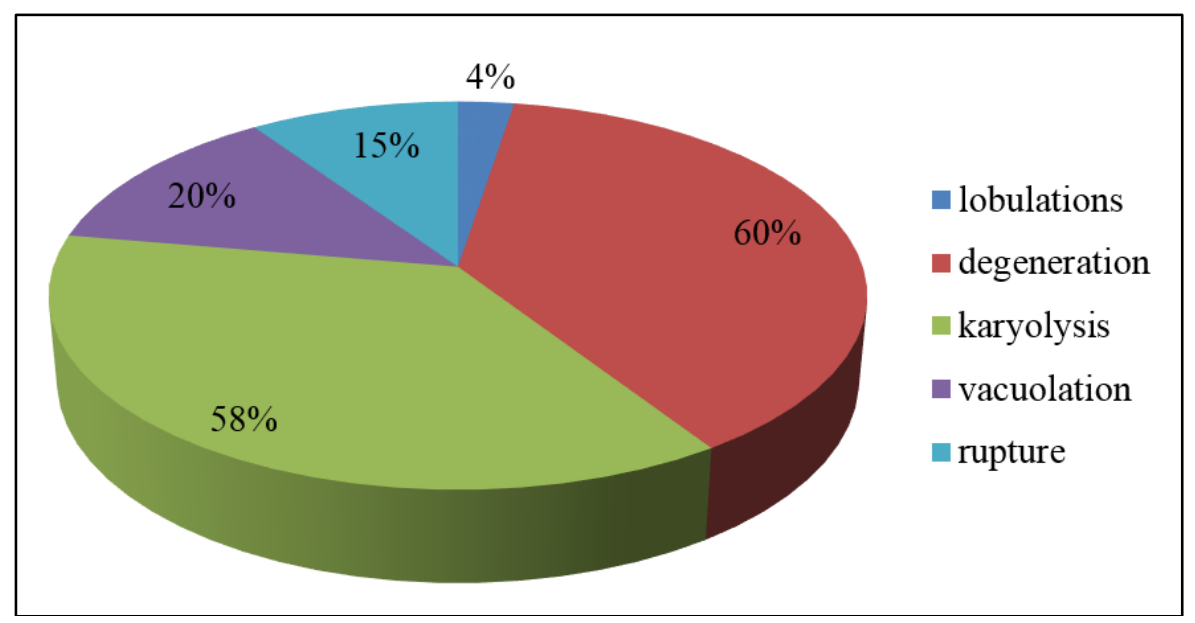

Graph 2: Graphical representation of EDTA induced cytoplasmic changes
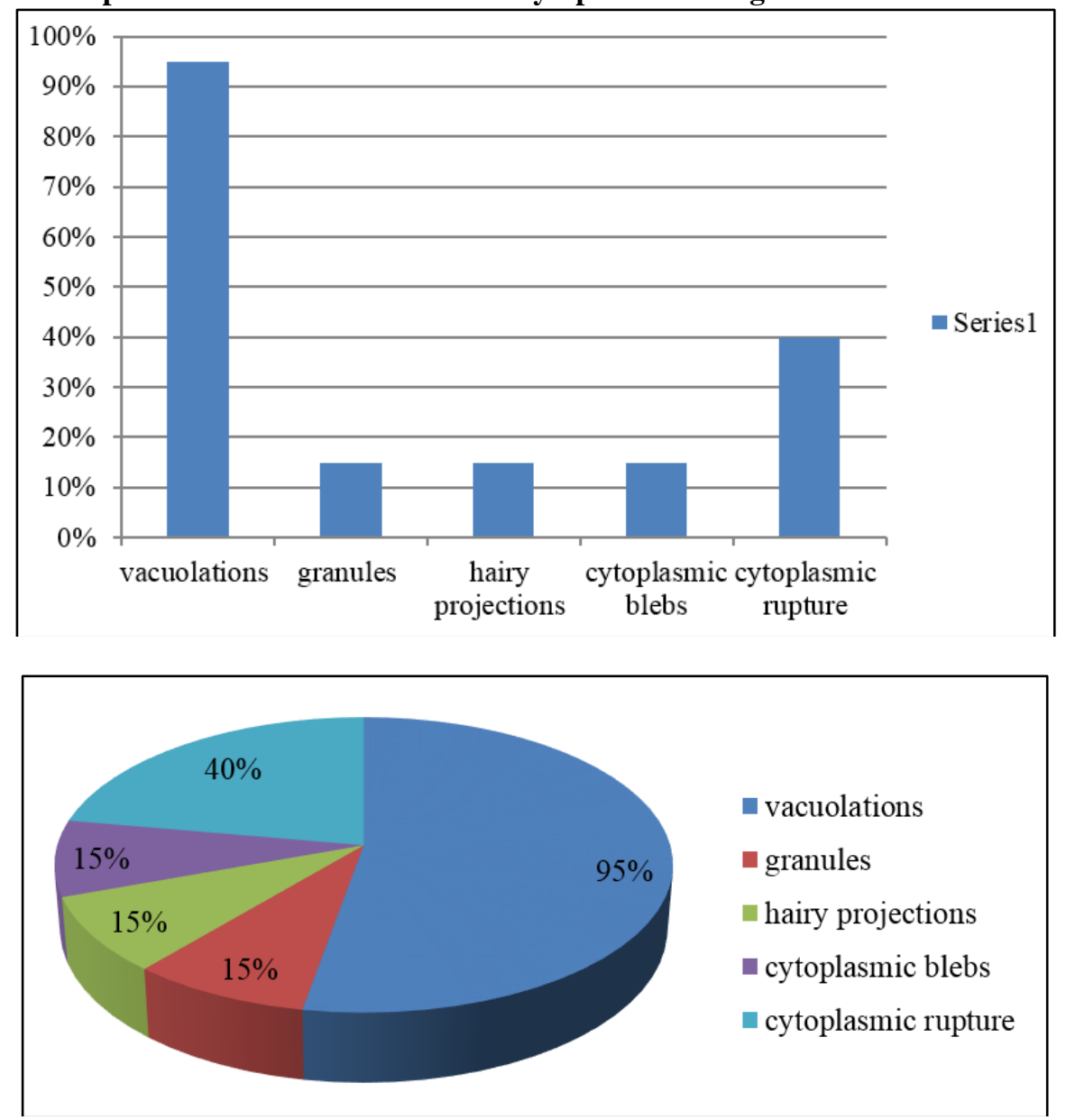

Storage artefacts misierpretated on peripheral blood smear:

WBC Storage Artefacts:

1. Neutrophil-nuclear lobulation and cytoplasmic vacuolation $\rightarrow$ Infection/sepsis

2. Lymphocyte - nuclear lobulation $\rightarrow$ Hyperchromatic nuclei

3. Monocytes -cytoplasmic vacuolation and nuclear degeneration $\rightarrow$ Infection/sepsis

\section{Platelet Storage Artefacts:}

1. Platelet aggregation $\rightarrow$ Pseudothrombocytopenia RBC Storage Artefacts:

1. Crenated $\mathrm{RBC} \rightarrow$ Burr cells

2. Loss of central pallor $\rightarrow$ Spherocyte

Therefore while examination of peripheral blood smears all these changes should be kept in mind so that they are not misinterpreted as pathological findings. 


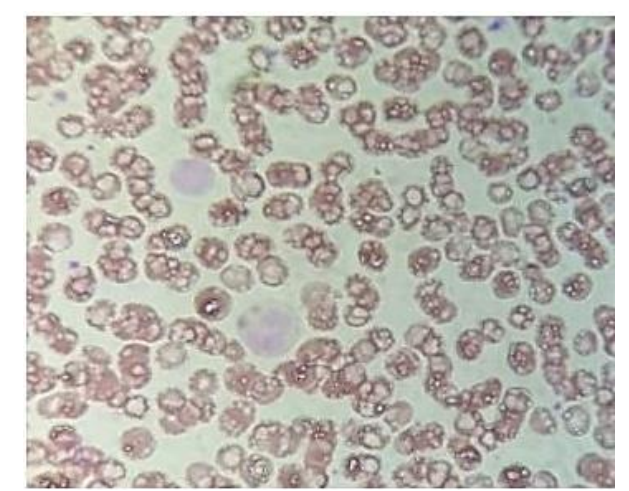

Fig. 1: Abnormal staining with crenated RBCs-1

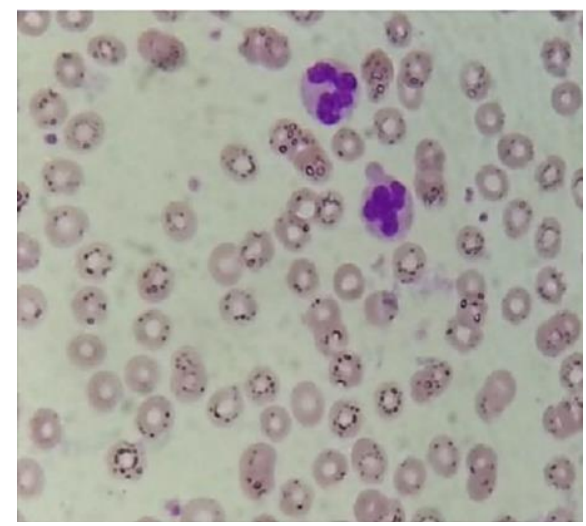

Fig. 2: Cytoplasmic blebs-1

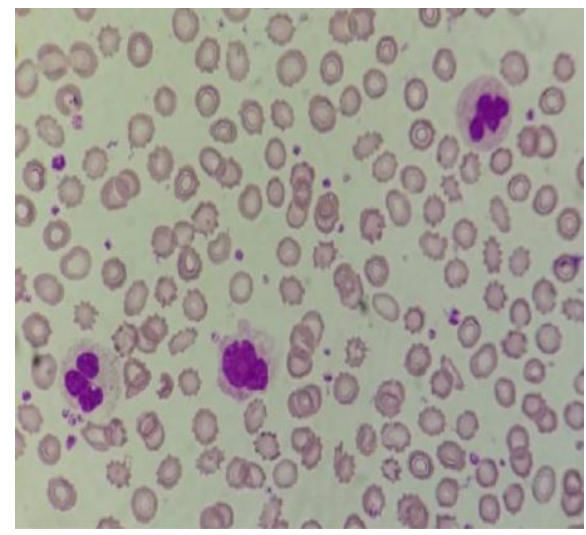

Fig. 3: Cytoplasmic vacuolation and nuclear pyknosis-1

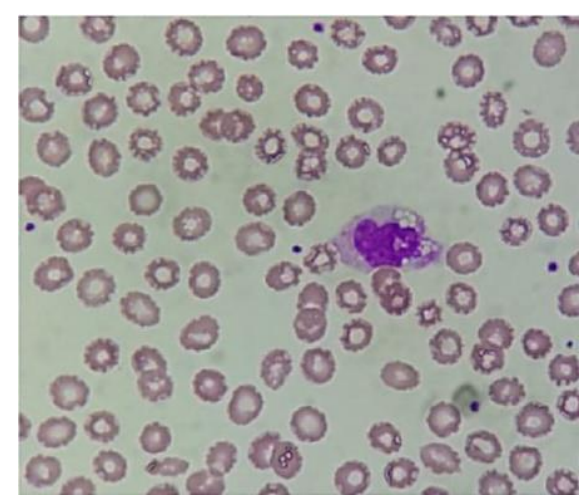

Fig. 4: Cytoplasmic vacuolation-1

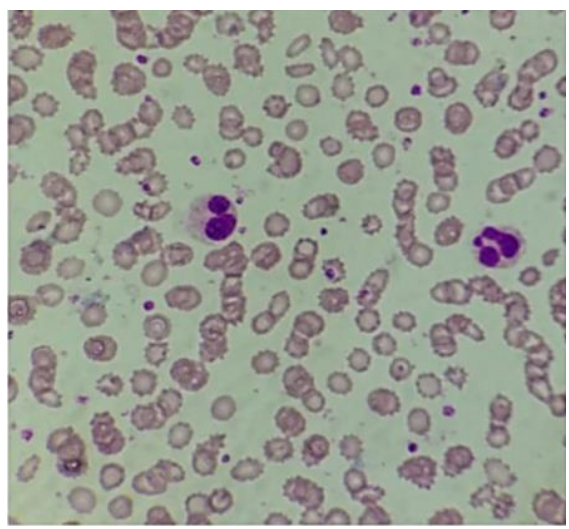

Fig. 5: Nuclear pyknosis-1

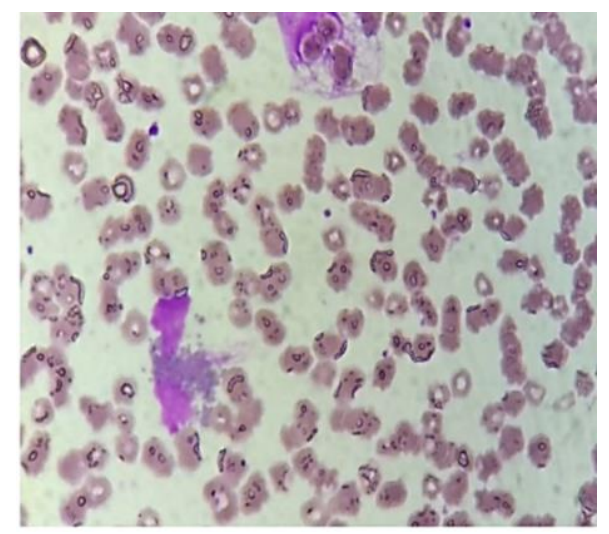

Fig. 6: Nuclear vacuolation-1

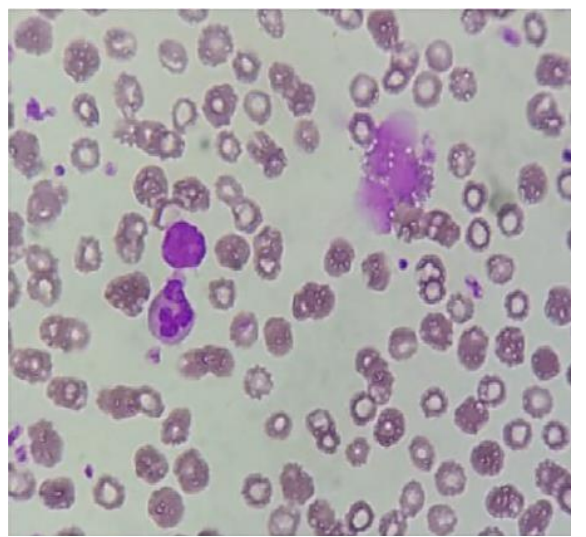

Fig. 7: Smudge cells-1

\section{Discussion}

Peripheral blood smear is an important and informative tool for screening, diagnosis and monitoring of disease. Morphological evaluation of peripheral blood smear provides an important clue as many diseases manifest with changes in peripheral blood. ${ }^{1,5}$ EDTA is the preferred anticoagulant for automated blood cell counts. However it causes morphological alterations on prolonged storage leading to erroneous diagnosis. ${ }^{6}$ The finger prick smears which served as controls showed only platelet aggregation. Smears studied immediately after addition of anticoagulant did not show any artefactual changes. As 
the EDTA blood stands in the test tube changes in leukocyte morphology begin to take place. The need of the hour is to have knowledge about the artifactual changes which can mimick various mild to serious disorders hence giving false/biased information to the clinician. And therefore clinical history must be asked for in cases where it is not provided especially when one comes across deviation from normal in case of morphology of blood components like RBC's, WBC's and platelets. In the present study we came across various artifactual changes.

Following changes were observed:

Nuclear Changes: Initially lobulations were observed as early as $2 \mathrm{hrs}$ followed by nuclear degeneration, karyolysis, nuclear vacuolations and nuclear rupture which began as early as 0hrs with citrate. Similar findings were noted by Koolwal, Vajpayee and Raphael et al. ${ }^{7,9,10}$

Cytoplasmic Changes: cytoplasmic vacuoles began to occur as early as 0hrs with sodium citrate with completely altered morphology including hairy projections, blebs and rupture at the end of 6hrs. The changes are delayed upto $2 \mathrm{hrs}$ with EDTA blood.

Platelets: Swelling of platelets occure as early as $2 \mathrm{hrs}$ with EDTA and $1 \mathrm{hr}$ with citrate. EDTA induced pseudo - agglutination of platelets initiated at $3 \mathrm{hrs}$ and marked at $6 \mathrm{hrs}$ no significant platelet aggregation was observed with citrate blood. The findings correlates with Koolwal and Chavda et al. ${ }^{7,8}$

Other artefacts like smudge cells, increase in size of WBCS, crenated RBC's and abnormal staining were also observed which began as early as $1 \mathrm{hr}$ with citrate but delayed upto 3-4hrs with EDTA blood. Similar findings were noted by Narasimha et al. ${ }^{11}$

All these changes can be misinterpreted as pathological findings. With respect to WBC morphology vacuolisation of monocytes is observed. Neutrophils often show separation of nuclear lobes and cytoplasmic vacuolation. Cytoplasmic vacuolation of neutrophils mimicks infection/sepsis. Where as lymphocytes show nuclear lobulation which can be misinterpreted as malignant cells. ${ }^{5}$ EDTA samples sometimes may lead to pseudo thrombocytopenia. This may be due to poor mixing of blood with EDTA or due to insufficient EDTA. ${ }^{12} 0.1 \%$ of individuals have EDTA - dependent agglutinins which can induce platelet clumping.

\section{Conclusion}

The observations of our study show that marked changes occur in RBC, WBC and platelet morphology if the blood samples collected in EDTA anticoagulant are stored over a period of time, which may result in misinterpretation leading to wrong diagnosis. EDTA has been recommended as the anticoagulant of choice for peripheral blood smear as it allows preservation of cellular components and does not alter the cellular morphology. ${ }^{12}$ Citrate should be avoided as it may result in increase in cell lysis and altered morphology. ${ }^{9,10}$ Therefore it is recommended that analysis of PBS should be made with in $1 \mathrm{hr}$ of collection which is permissible with EDTA blood but not beyond. ${ }^{8,11}$

\section{References}

1. Sathe Jayashree, Pramanik Sanjay S.EDTA induced morphological changes in the cells of peripheral blood smear. International Journal of Recent Trends in Science and Technology. 2017;22(2):111-113.

2. McNamara C, J. Bain B. Dacie and Lewis Practical Haematology. 12th ed. Elseveir; 2017.

3. Smock KJ, Perkins SL. Examination of the blood and bone marrow. In Wintrobe's Clinical Hematology: Thirteenth Edition. Wolters Kluwer Health Adis (ESP). 2013. p. 1-18.

4. Narasimha A, Kumar H, Prasad C. Anticoagulant induced artefacts in peripheral blood smears. Indian Journal of Hematology and Blood Transfusion. 2008;24(2):43-48.

5. Zini G. Stability of complete blood count parameters with storage: toward defined specifications for different diagnostic applications. International Journal of Laboratory Hematology. 2013;36(2):111-113.

6. Antwi-Baffour S. Prolong Storage of Blood in EDTA Has an Effect on the Morphology and Osmotic Fragility of Erythrocytes. International Journal of Biomedical Science and Engineering. 2013;1(2):20.

7. Koolwal D. Anticoagulant EDTA Induced Storage effect (Artifacts) on Peripheral Blood Cells. Journal of Medical Science and clinical Research. 2018;6(1).

8. Chavda D, Mehta D. Storage artifacts in peripheral blood smears. Indian Journal of Basic and Applied Medical Research. 2016;5(2):8-12.

9. Vajpayee N, Graham SS, Bem S (2007) Basic Examination of Blood and Bone Marrow, In: McPherson RA, Pincus MR, Eds. Henry's Clinicl Diagnosis and Management by Laboratory Methods. (21st Edition. India: Saunders, 476.

10. Raphael SS (1983) Principles of Haematology in: Raphael SS, Eds. Lynch's Medical Laboratory Technology. 4th edition. Philadelphia: Saunders 126.

How to cite this article: Choudhary S, Katkar RS, Nagaram D. Storage artefacts in peripheral blood smears. J Diagn Pathol Oncol. 2018;4(3):187-191. 